\title{
Exuberant inflammatory reaction after an infiltration of platelet-rich plasma (PRP)
}

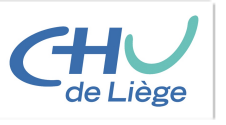

$$
\underset{\text { Kaux JF }}{\text { University and University Hospital of Liège, Belgium }}
$$

Background: PRP, obtained from centrifuged autologous blood, contains a large quantity of growth factors, which may enhance the tissue healing processes. Local infiltration of PRP represents a relatively new treatment for tendinopathies. To date, no side effects have been reported after infiltration of PRP to treat tendinopathy ${ }^{1}$.

Case report: A 35-year-old patient had a right upper patellar tendinopathy which was resistant to all conservative treatments for more than 6 months. The patient was a type 1 diabetic (well controlled). He had an intratendinous infiltration of $6 \mathrm{~mL}$ of PRP $\left(8.10^{5}\right.$ platelets $/ \mathrm{mm}^{3}$, almost no red or white blood cells $)$ after disinfection but without local anaesthetic. Immediately following the infiltration, local cryotherapy was performed for 15 minutes. NSAIDs were avoided, but class-1 or -2 pain-killers were authorised if necessary. A standardised submaximal eccentric rehabilitation should have been started 1 week after. However, the patient experienced local swelling with erythema, increased heating and pain which appeared just underneath the patella, but without biological inflammatory syndrome. A great Doppler signal in a thicker patellar tendon was observed by US, but there was no sign of local infectious disease demonstrated by either CT or MRI. However, the local inflammation did not decrease after a progressive 3-week treatment of local cryotherapy, local and oral NSAIDs and colchicine $1 \mathrm{mg}$. Thus, an insidious infection was suspected, even though there was neither evidence of biological inflammatory syndrome nor sign of infectious lesion on imagery examination. An antibiotic therapy (rifampicine $600 \mathrm{mg}+$ minocycline $100 \mathrm{mg}$ ) was initiated for 3 months. Finally, a 3-phase bone scintigraphy suggested the presence of a complex regional pain syndrome type 1 treated by a classical physical therapy and concomitant class-2 pain killers. The evolution was favourable after 6 months of symptomatic treatment, and the pain decreased to a level similar to that before the infiltration of PRP.

Discussion/Conclusions: This case report draws attention to potential side effects that are linked to this new therapy by infiltration of platelet rich plasma in case of tendinopathy, in particular when used in patients with type 1 diabetes. Thus, the balance between benefits and risks must be carefully evaluated before using this treatment in patients with type 1 diabetes. 